\title{
¿Es necesario el Axioma de Zermelo para comprender la teoría de la medida?*
}

\section{Is Zermelo's Axiom Necessary for Understanding Measure Theory?}

\author{
Carmen Martínez-Adame $e^{\dagger \ddagger}$
}

\begin{abstract}
Resumen
En este artículo estudiamos la relación que guardan entre sí el axioma de elección y la teoría de la medida y analizamos el papel que tiene el axioma para una comprensión plena de la teoría. El tema de la comprensión en matemáticas es muy extenso y ha sido ampliamente estudiado desde diversos puntos de vista; nosotros pretendemos abordar este tema desde dentro de la matemática misma.
\end{abstract}

Palabras clave: axioma de elección - principio del buen orden - conjuntos medibles - fundamentos de las matemáticas

\begin{abstract}
In this paper we study the relation between the axiom of choice and measure theory and we analyze the role that the axiom plays in obtaining a full understanding of the theory. Comprehension in mathematics is an extensive subject that has been widely studied from many points of view; our approach here will come from within mathematics itself.
\end{abstract}

Keywords: axiom of choice - well-ordering principle - measurable sets - foundations of mathematics

\footnotetext{
* Recibido: 25 de Junio de 2012. Aceptado en versión revisada: 27 de Septiembre de 2012.

${ }^{\dagger}$ Departamento de Matemáticas, Facultad de Ciencias, Universidad Nacional Autónoma de México, México D.F. Para contactar a la autora, por favor, escriba a: cmai@matematicas.unam.mx.

Metatheoria 3(2)(2013): 37-64. ISSN 1853-2322.

(C) Editorial de la Universidad Nacional de Tres de Febrero. Publicado en la República Argentina.
} 


\section{Introducción}

El Axioma de Elección tiene una historia larga y muy interesante; no obstante, la cuestión que nos interesa en este artículo es su relación con la teoría de la medida y en particular el papel que juega este axioma para llegar a comprender plenamente esta teoría. Este axioma se puede enunciar, como ya es sabido, de muchas formas; destacamos aquí algunas de las más usuales: Si T es un conjunto cuyos elementos son todos conjuntos disjuntos y distintos del conjunto vacío, entonces su unión $\mathfrak{S} T$ incluye al menos un subconjunto $S_{1}$ que tiene uno y solo un elemento en común con cada elemento de $T$ (Zermelo 1908b); para toda familia $F$ de conjuntos no vacíos, hay una función $f$ tal que $f(S)$ Î $S$ para cada conjunto $S$ en la familia $F$ (Jech 1973), o bien, para cada familia $\left(\chi_{i}\right)_{i \text { iI }}$ de conjuntos no vacíos $\chi_{i}$, el conjunto producto $\prod_{i \hat{i I l}} \chi_{i}$ es no vacío (Herrlich 2006). ${ }^{1}$

El estudio que llevaremos a cabo en este artículo permitirá un análisis de la relación entre el axioma de elección y la teoría de la medida en la misma línea en la que en 1943 Stanislaw Ulam afirmaba lo siguiente:

La creación de Cantor de la teoría de conjuntos en la que la noción de figura geométrica se generalizó a la de un subconjunto arbitrario de puntos de un espacio dado, también introdujo la necesidad de una investigación axiomática del problema de la medida de conjuntos y, al mismo tiempo, hizo posible un análisis lógico de la noción de medida en general. (Ulam 1943, p. 597)

Para poder llevar a cabo esta investigación es necesario estudiar el surgimiento del axioma de elección, su aceptación y su uso así como el surgimiento de la teoría de la medida y su desarrollo dentro de las matemáticas.

Creemos que es importante señalar que el objetivo de este artículo es estudiar el papel que el axioma de elección juega en la comprensión de la teoría de la medida y en particular estudiaremos su rol en la evolución del problema de la medida. Pretendemos mostrar que aunque se puede argumentar que es precisamente el resultado de Solovay (1970) el que muestra de manera sistemática la conexión que existe entre el axioma de elección y la medida de Lebesgue ${ }^{2}$ esta relación era ya evidente para 1930.

\section{El Axioma de Zermelo}

Ernst Zermelo llegó a Göttingen en noviembre de 1897 y, bajo la influencia de David Hilbert, pronto comenzó a trabajar sobre cuestiones relativas a los fundamentos de las matemáticas. Antes de dedicarse a estas cuestiones, Zermelo se dedicaba primordialmente a las matemáticas aplicadas pero fue su trabajo funda-

\footnotetext{
${ }^{1}$ Es importante recordar que Gödel (1940) mostró que la negación del axioma de elección no es un teorema de la teoría de conjuntos (ZF) y que Cohen (1963) mostró que el axioma de elección tampoco es un teorema de ZF, mostrando así su independencia. Cabe mencionar que estos resultados son posteriores a los resultados que analizaremos para tratar de dar respuesta a la pregunta que intitula a este artículo.

${ }^{2}$ En este artículo, Solovay exhibe un modelo de la teoría de conjuntos (donde no se satisface el axioma de elección) en el cual cualquier conjunto de números reales es medible en el sentido de Lebesgue.
} 
cional, y en particular su trabajo sobre la teoría de conjuntos, el que lo llevó a alcanzar sus logros más destacados.

En 1900 se celebró en París el Segundo Congreso Internacional de Matemáticas y el 8 de agosto Hilbert presentó, en su conferencia en la Sorbonne, su famosa lista de 23 problemas abiertos en matemáticas con los que dictaba a la comunidad matemática la agenda para el nuevo siglo. Durante la conferencia, por cuestiones de tiempo, Hilbert únicamente enunció 10 de estos problemas (1, 2, $6,7,8,13,16,19,21$ y 22), pero el texto completo, Mathematische Probleme, fue publicado ese mismo año. ${ }^{3}$ Los primeros seis problemas tienen que ver con cuestiones fundacionales y se pueden considerar la base para el programa que seguiría la investigación sobre los fundamentos de las matemáticas en Göttingen.

El primer problema es "el problema de Cantor" sobre el número cardinal del continuo", que textualmente dice:

Se dice (siguiendo a Cantor) que dos sistemas, i.e., dos conjuntos de números reales ordinarios o puntos son equivalentes o tienen número cardinal igual si pueden ser puestos en relación uno con el otro de manera que a cada número de un conjunto corresponda uno y solo un número definido del otro. Las investigaciones de Cantor sobre tales conjuntos sugieren un teorema muy plausible, que no obstante los más grandes esfuerzos nadie ha podido demostrar. Este es el teorema:

Todo sistema con una cantidad infinita de números reales, i.e., todo conjuntos de números (o puntos), es o bien equivalente al conjunto de enteros naturales $1,2,3, \ldots$ o al conjunto de todos los números reales y por tanto al continuo, es decir, a los puntos de una línea; por tanto, en cuanto a equivalencias, sólo hay dos conjuntos de números, el conjunto numerable y el continuo.

A partir de este teorema se seguiría inmediatamente que el continuo tiene el número cardinal siguiente al del conjunto numerable; la demostración de este teorema formaría por tanto un nuevo puente entre el conjunto numerable y el continuo.

Déjenme mencionar otro notable enunciado de Cantor que guarda una muy estrecha relación con el teorema mencionado y que quizás ofrece la cla-

\footnotetext{
${ }^{3}$ En 1900 en Nachrichten von der Königlcihen Gesellschaft der Wissenschaften zu Göttingen (pp. 253-297). También fue publicado en 1901 en el Archiv der Mathematik und Physik (3)(1): 44-63 y 213-237. El discurso fue traducido al inglés y publicado en 1902 en el Bulletin of the American Mathematical Society 8: 437-479, y la traducción al francés Sur les problèmes futurs des mathématiques apareció publicada en 1902 en Compte Rendu du Deuxième Congrès International des Mathématiciens, pp. 58-114.

${ }^{4}$ Este problema lo llama Hilbert el problema de Cantor, pues fue Georg Cantor quien primero enunció el resultado que Hilbert pide probar que se conoce como la hipótesis del continuo: Todo subconjunto infinito de $\mathrm{R}$ es numerable o bien tiene la potencia del continuo c. Este resultado aparece publicado (sin demostración, evidentemente) en Cantor (1878). Utilizando la notación de los alephs este problema se vuelve: No existe $m$ tal que $\boldsymbol{N}_{0}<m<c$. En una carta a Dedekind, fechada en noviembre de 1882 (publicada en Cavaillès 1994 y traducida al español en Cantor 2006), Cantor afirma que $\mathrm{R}$ tiene la potencia de la segunda clase de números transfinitos, es decir el conjunto de todos los ordinales numerables. O lo que es lo mismo (utilizando la notación de los alephs): que no existe $m$ tal que $\aleph_{0}<m<c \aleph_{1}$, es decir, $2^{\aleph_{0}}=\aleph_{1}$. Este segundo enunciado es más fuerte que el primero, hecho que resulta relevante para nosotros puesto que este enunciado implica que $\mathrm{R}$ se puede bien ordenar pero el primero no. Es más, el segundo es equivalente a la conjunción del primero y la existencia de un buen orden para R. Remitimos al lector interesado a Sierpinski (1934), Godel (1947), Álvarez (1993) y Moore (2002), donde puede obtener una historia detallada de los orígenes de la hipótesis del continuo.
} 
ve para su demostración. Cualquier sistema de números reales se dice ordenado si para cualesquiera dos números del sistema se puede determinar cuál ocurre antes y cuál después y si al mismo tiempo esta determinación es tal que $a$ es antes que $b$ y $b$ es antes que $c$, entonces $a$ es antes que $c$. El arreglo natural de los números en un sistema se define como aquel en el que lo más pequeño precede a lo más grande, pero se puede ver fácilmente que hay una infinidad de maneras en las cuales el sistema se puede acomodar.

Si pensamos en un arreglo de números definido y seleccionamos un sistema particular de estos números, un llamado sistema parcial, este sistema parcial también estará ordenado. Cantor considera un tipo particular de conjunto ordenado que designa como conjunto bien ordenado y que se caracteriza por el hecho de que en cualquier conjunto parcial, como en el conjunto mismo, existe un primer número. El sistema de enteros $1,2,3, \ldots$ en su orden natural es evidentemente un conjunto bien ordenado. Por otro lado, el sistema de todos los números reales, i.e., el continuo con su orden natural, evidentemente no es bien ordenado. Si pensamos en los puntos de un segmento de línea recta, con su punto inicial excluido, como nuestro conjunto parcial, este no tendrá primer elemento.

El problema que surge es si la totalidad de números puede ser acomodada de otra manera para que cualquier conjunto parcial tenga un primer elemento, i.e., si el continuo se puede considerar como un conjunto bien ordenado - una cuestión que Cantor piensa que debe ser contestada de manera afirmativa-. A mí me parece muy deseable obtener una prueba directa de este notable enunciado de Cantor, quizás mediante el dar un arreglo para los números de manera que en cada sistema parcial se pueda señalar un primer elemento. (Hilbert 1900, pp. 263-264)

El segundo problema trata de la compatibilidad de los axiomas de la aritmética, es decir, la consistencia de los axiomas para los números reales. La solución de este problema sería demostrar que estos axiomas no son contradictorios, es decir, que un número definido de pasos lógicos basados en ellos nunca puede llevar a resultados contradictorios. Ahora bien, un hecho clave es que, a partir de esta no contradicción, Hilbert asegura que se puede concluir la existencia matemática del sistema completo de los números reales, puesto que, para él, la existencia en matemáticas se demuestra precisamente a partir de la no contradicción: "Si a un concepto se le asignan atributos contradictorios yo digo que el concepto no existe matemáticamente [...]. Pero si se puede demostrar que los atributos asignados a un concepto nunca llevarán a una contradicción a través de la aplicación de un número finito de procesos lógicos, entonces digo que se ha demostrado la existencia matemática del concepto" (Hilbert 1900, [Problema 2], p. 265).

\footnotetext{
${ }^{5}$ Esta segunda parte del problema de Hilbert también tiene sus orígenes en un enunciado de Cantor (como se puede apreciar por el texto mismo). En 1883 Cantor enuncia por primera vez lo que llama una ley del pensamiento: Todo conjunto puede ser bien ordenado (traducido al español en Cantor 2006). Al afirmar que es una ley del pensamiento Cantor implica que es autoevidente este resultado, que se conoce como el principio del buen orden. Posteriormente, alrededor de 1895, Cantor cambia de parecer y afirma que este principio no es una ley autoevidente del pensamiento y que requiere de una demostración. Incluso en 1897 creyó haber encontrado una demostración pero él mismo se dio cuenta de que la prueba no era correcta y no permitió que se publicara. Sin embargo, fue solo con este primer problema de Hilbert que el enunciado de Cantor cobró un interés mayor.
} 
Hilbert argumenta que esta prueba de existencia a través de una axiomatización consistente también debería ser posible para los cardinales y las clases de números de Cantor pero no para la totalidad de números cardinales o de alephs "para los cuales, como se puede mostrar, un sistema de axiomas, consistente en mi sentido, no puede ser establecido. Cualquiera de estos sistemas es, por tanto, de acuerdo con mi terminología, matemáticamente inexistente" (Hilbert 1900, [Problema 2], p. 266).

En la última parte de la década de 1890, Hilbert y Cantor sostuvieron una correspondencia ${ }^{6}$ sobre temas relacionados con la teoría de conjuntos en la cual se dio particular importancia a la existencia de conjuntos paradójicos, o de las multiplicidades inconsistentes en la terminología cantoriana, como el conjunto de todos los alephs. En un primer momento, estos problemas se consideraban en Göttingen como particulares de la teoría de conjuntos en tanto que una rama de las matemáticas y fue la publicación en 1903 de la paradoja de Russell (como se la conoce ahora) lo que llevó a mostrar que estas paradojas presentaban un fuerte problema para el programa de Hilbert.

En una carta a Frege fechada el 16 de junio de $1902,{ }^{7}$ Russell escribe su paradoja: "no hay clase (como totalidad) de las clases que al ser tomadas cada una como totalidad no pertenecen a ellas mismas" (Frege 1976, p. 211). Tal clase pertenecería y no pertenecería a la vez a sí misma.

Hilbert se enteró de esta paradoja a través del segundo volumen de los Grundgesetze ${ }^{8}$ de Frege que él mismo le había enviado. Hilbert le contestó a Frege que este ejemplo se conocía ya en Göttingen y que había sido Zermelo, hacía tres o cuatro años, quien lo había descubierto. ${ }^{9}$ Vale la pena notar que, en su artículo de 1908 sobre el teorema del buen orden, Zermelo mismo afirma haber encontrado la paradoja de forma independiente a Russell y habérsela comentado a Hilbert antes de $1903 .^{10}$

Dado esto, en 1903 se volvió evidente que se necesitaba un trabajo sobre los fundamentos de las matemáticas; este trabajo se vio íntimamente relacionado con el trabajo de Zermelo en la teoría de conjuntos.

\footnotetext{
${ }^{6}$ Un análisis detallado de esta correspondencia se puede encontrar en Purkert (1987) y en Cantor (2006).

${ }^{7}$ Una traducción al inglés de esta carta se encuentra publicada en van Heijenoort (1967).

${ }^{8}$ Véase Frege (1903).

${ }^{9}$ En 1905, Hilbert (Hilbert 1905, p. 210) presentó la paradoja de Russell como una paradoja puramente lógica que probablemente sería más convincente para los no matemáticos, argumentando que él mismo había formulado una que resultaba más pertinente para los matemáticos. Para construir esta paradoja, Hilbert contempla dos principios para formar conjuntos: el principio de suma (que permite la unión de conjuntos hasta en cantidades infinitas) y el principio del mapeo (que, para un conjunto dado $S$, permite formar el conjunto $S^{S}$ de todos los mapeos de $S$ en sí mismo). Hilbert considera todos los conjuntos que resultan al aplicar estos principios un número arbitrario de veces al conjunto de los números naturales. Usando ambos principios nuevamente se obtiene la unión $U$ de todos estos conjuntos y el conjunto correspondiente $U^{U}$. Por definición, el conjunto $U^{U}$ es un subconjunto de $U$. Sin embargo, como la cardinalidad de $U^{U}$ es estrictamente mayor a la de $U$, esto produce una contradicción.

${ }^{10}$ Véase Zermelo (1908a). Vale la pena notar también que entre los documentos de Edmund Husserl se encontró una nota manuscrita diciendo que Zermelo le había informado de una paradoja en la teoría de conjuntos en abril de 1902. Véase Husserl (1979).
} 
En 1900 Zermelo dictó lo que se cree es el primer curso en la historia dedicado por completo a la teoría de conjuntos y resulta relevante que un primer resultado que Zermelo probó en 1901 es que si $\mu$, un cardinal, permanece invariante al serle sumado cualquier cardinal de la sucesión infinita $\boldsymbol{\aleph}_{1}, \boldsymbol{\aleph}_{2}, \boldsymbol{\aleph}_{3}, \ldots$ entonces permanece invariante también cuando le son sumados todos a la vez. Es importante notar que el argumento de Zermelo hace un uso tácito del Axioma de Elección (tres años antes de enunciarlo explícitamente por primera vez).

Poco tiempo después, en 1904, se llevó a cabo el Tercer Congreso Internacional de Matemáticas en Heidelberg y, en el marco de este congreso, el 9 de agosto, ante la presencia de Cantor y de Hilbert, Gyula König ${ }^{11}$ dictó una conferencia sobre el problema del continuo (el primer problema planteado por Hilbert cuatro años antes) en la cual afirmaba haber demostrado que la cardinalidad del continuo no era ni un aleph ni la cardinalidad de un conjunto bien ordenado, es decir, que la conjetura de Cantor era falsa. Por tanto también afirmaba haber probado que el continuo no se podía bien ordenar (segunda parte del primer problema de Hilbert).

En la primera parte de la conferencia, König demostró que para un cardinal $\boldsymbol{\aleph}_{\eta}$, supremo de un conjunto numerable de cardinales más pequeños, es decir, para un cardinal $\boldsymbol{\aleph}_{\eta}$ de cofinalidad $\omega$, el cardinal $\boldsymbol{\aleph}_{\eta}^{\boldsymbol{x}_{0}}$ del conjunto de funciones de $\boldsymbol{\aleph}_{0}$ a $\boldsymbol{\aleph}_{\eta}$ es más grande que $\boldsymbol{\aleph}_{\eta} \cdot{ }^{12}$ Luego, si se supone que la potencia $2^{\boldsymbol{\aleph}_{0}}$ del continuo es un aleph, digamos $\boldsymbol{\aleph}_{\beta}$, y tomando como $\boldsymbol{\aleph}_{\eta}$ al cardinal $\boldsymbol{\aleph}_{\beta+\omega}$, es decir, al supremo de los cardinales $\boldsymbol{\aleph}_{\beta}, \boldsymbol{\aleph}_{\beta+1}, \boldsymbol{\aleph}_{\beta+2}, \ldots$ de cofinalidad $\omega$, König usó un resultado de Felix Bernstein ${ }^{13}$ para mostrar que $\boldsymbol{\aleph}_{\beta+\omega}^{\boldsymbol{K}_{0}}=\boldsymbol{\aleph}_{\beta+\omega} \cdot \boldsymbol{\aleph}_{\beta}$ y por tanto que $\boldsymbol{\aleph}_{\beta+\omega}^{\aleph_{0}}=\aleph_{\beta+\omega}$ contradiciendo así su desigualdad. ${ }^{14}$

Kowalewski ${ }^{15}$ afirma que, al día siguiente, Zermelo encontró el error en la demostración de König, aunque Grattan-Guinness y Purkert cuestionan que Zermelo haya jugado un papel tan decisivo y sostienen que fue Hausdorff quien tiempo después del congreso encontró las fallas en la demostración. No parecen ser contradictorias estas dos posturas y no tomaremos partido por ninguna de ellas, aunque en general se considera que la postura de Kowalewski es menos fiable. Lo que sí es un hecho indiscutible es que existe un error en la demostración de König y que este radica en el uso del resultado de Bernstein, puesto que $\boldsymbol{\aleph}_{\alpha}^{\boldsymbol{N}_{0}}=\boldsymbol{\aleph}_{\alpha} \cdot 2^{\boldsymbol{\aleph}_{0}}$ no se cumple para cardinales de cofinalidad $\omega$.

De esta manera, la refutación de la hipótesis del continuo había sido ella misma refutada y, por ende, tanto ella como el principio del buen orden permanecían problemas abiertos.

\footnotetext{
${ }^{11}$ König era de nacionalidad húngara pero cuando publicaba algún texto en revistas alemanas lo hacía bajo el nombre de Julius König.

${ }^{12}$ Este resultado se conoce como la desigualdad de König.

${ }^{13}$ El resultado en cuestión fue demostrado por Bernstein en su tesis doctoral (1901) y afirma que $\aleph_{\alpha}^{\aleph_{0}}=\aleph_{\alpha} \cdot 2^{\boldsymbol{\aleph}_{0}}$ para todo ordinal $\alpha$.

${ }^{14}$ La demostración se puede consultar en König (1905) y un análisis detallado de esta se encuentra en Moore (1982).

${ }^{15}$ Véase Kowalewski (1950).
} 
Un mes después del congreso en Heidelberg Zermelo escribió una carta (fechada el 24 de septiembre de 1904) a Hilbert en la cual probaba el principio del buen orden. Hilbert publicó inmediatamente las partes relevantes de la carta en los Mathematische Annalen (Zermelo 1904). ${ }^{16}$

La demostración de Zermelo (1904) toma un conjunto arbitrario no vacío M al cual se le quiere dar un buen orden:

(1) Sea $M$ un conjunto arbitrario de cardinalidad $\boldsymbol{m}$, sea $m$ un elemento arbitrario de él, sea M', de cardinalidad 'm', un subconjunto de M que contiene por lo menos un elemento $m$ y que incluso puede contener a todos los elementos de $\mathrm{M}[\ldots]$. Sea $\mathcal{M}$ el conjunto de todos los subconjuntos de M'.

(2) Imaginemos que a cada subconjunto $\mathrm{M}$ ' se asocia un elemento arbitrario $m_{1}^{\prime}$ de M' mismo; llamemos a m' el elemento distinguido de $\mathrm{M}^{\prime}$. Esto produce una cubierta $^{17} \mathrm{~g}$ del conjunto $\mathrm{M}$ por ciertos elementos del conjunto $\mathrm{M}[\ldots]$. (Zermelo 1904, p. 514).

Utilizando como principal herramienta la frase con la cual comienza (2), es decir, el primer enunciado explícito de lo que más tarde se llamaría el Axioma de Elección, Zermelo probó el principio del buen orden.

Esta prueba suscitó muchas discusiones, no tanto relacionadas con el resultado sino con el método utilizado por Zermelo para llegar a él. Según Zermelo la demostración estaba basada sobre un "principio lógico que no puede ser reducido a uno más simple pero que es aplicado sin vacilación en todas partes en las deducciones matemáticas" (Zermelo 1904, p. 516).

Cuatro años después de la publicación de la prueba del principio del buen orden, Zermelo publicó un artículo nuevo (Zermelo 1908a), en el cual proporciona una nueva demostración del mismo principio y defiende el uso del axioma de elección. Para demostrar en parte la indispensabilidad de dicho axioma enuncia siete teoremas ${ }^{18}$ bien conocidos en cuyas demostraciones el axioma es necesario. Estos teoremas incluyen los siguientes resultados:

(i) Si un conjunto $\mathrm{M}$ se puede descomponer en partes disjuntas $\mathrm{A}, \mathrm{B}, \mathrm{C}, \ldots$ el conjunto de estas partes es equivalente a un subconjunto de $M$.

(ii) Las uniones disjuntas de conjuntos equivalentes son equivalentes.

(iii) El producto de varias cardinalidades se puede anular solo si uno de los factores se anula.

(iv) Un conjunto que no es equivalente a ninguna de sus partes siempre se puede ordenar de manera que cada subconjunto tenga un primer y un último elemento.

(v) La unión numerable de conjuntos numerables es numerable.

\footnotetext{
${ }^{16}$ Una traducción al inglés de esta carta se encuentra publicada en van Heijenoort (1967).

${ }^{17}$ Es conveniente precisar que Zermelo se refiere a una aplicación no inyectiva al utilizar la palabra cubierta, que en realidad ha tomado de Cantor.

${ }^{18} \mathrm{~A}$ estos siete resultados enunciados por Zermelo se pueden agregar muchos más, una buena referencia es Herrlich (2006).
} 
(vi) La existencia de una base de Hamel para el espacio vectorial de los números reales.

(vii) La existencia de soluciones discontinuas de la ecuación $f(x+y)=f(x)+f(y)$.

Vale la pena mencionar que el Axioma de Elección había sido utilizado antes de ser explícitamente enunciado por Zermelo sin llamar demasiado la atención, tanto en la teoría de conjuntos misma como en el análisis matemático. Algunos ejemplos son la demostración publicada en 1872 por Heine (Heine 1872) de un resultado de Cantor en el cual se afirma que la continuidad de una función real $f$ en un punto $x$ es equivalente a la continuidad secuencial de la función en el mismo punto. Otro ejemplo es el uso del hecho de que la unión numerable de conjuntos numerables es numerable, ${ }^{19}$ así como que todo conjunto (Dedekind-) infinito contiene un conjunto infinito numerable. ${ }^{20}$

También es interesante notar que, en 1890, G. Peano había negado explícitamente el principio usado por Zermelo: "[N]o se puede aplicar un número infinito de veces una ley arbitraria mediante la cual a una clase $a$ se le hace corresponder un individuo de esa clase" (Peano 1890, p. 210). Es interesante notar que este comentario aparece en un artículo dedicado a la integrabilidad de ecuaciones diferenciales en el cual Peano tenía interés de mostrar el uso de su simbolismo matemático y no en un texto dedicado enteramente a su cálculo lógico.

Ahora bien, dado esto, no es difícil comprender que Fraenkel y Bar-Hillel caractericen al axioma de elección como "el más interesante, y no obstante su tardía aparición, el axioma más discutido en matemáticas, después del axioma de Euclides de las paralelas que fue introducido hace más de dos mil años" (Fraenkel 1958, p. 47). Una clara muestra de la discusión en torno de este axioma se puede apreciar entre los analistas franceses de principios del siglo XX, como veremos a continuación.

\section{Cinco cartas sobre la teoría de conjuntos}

Después de la publicación en 1904 de la prueba del principio del buen orden, Hilbert (y la redacción de los Mathematische Annalen) le pide a Émile Borel unas reflexiones sobre la prueba. Borel considera un conjunto $M$ cualquiera e imagina dos problemas:

(i) Dar a M la forma de un conjunto bien ordenado.

(ii) Dado un subconjunto $M^{\prime}$ cualquiera de $M$, escoger en $M^{\prime}$ de manera determinada (pero por otra parte arbitraria) un elemento $m^{\prime}$, al cual se le dará el nombre de elemento distinguido de $M^{\prime}$ ', esta elección se deberá hacer para todos los subconjuntos M' de M.

\footnotetext{
${ }^{19}$ Este resultado se había utilizado de manera frecuente tanto en el análisis como en la teoría de conjuntos y podemos encontrar su origen en la demostración de la numerabilidad de los racionales dada por Cantor en 1870, aunque recién apareció publicado en 1878. Véase Cantor (1878).

${ }^{20}$ Véase Cantor (1895) y Borel (1898).
} 
Dados estos dos problemas dice:

Es evidente que toda solución del problema A proporciona una solución particular del problema B, pero el recíproco no es evidente y debemos al Sr. Zermelo el saber que los problemas A y B son equivalentes. Pero este resultado, cualquiera que sea su interés, no debe ser considerado como una solución general del problema A. (Borel 1904, p. 194)

Borel continúa diciendo que, para que se pueda considerar resuelto el problema $\mathrm{B}$, se debe dar un medio, al menos teórico, de determinar al elemento distinguido de cada subconjunto y dice que este problema parece extremadamente complicado si se supone que $\mathrm{M}$ es, por ejemplo, el continuo. Borel argumenta que el razonamiento dado por Zermelo es equivalente al siguiente: "Para bien ordenar al conjunto $M$ es suficiente escoger arbitrariamente un elemento al cual se le dará el rango 1, después otro al cual se le dará el rango 2, y así sucesiva y transfinitamente [...]. Ningún matemático verá como aceptable este razonamiento" (Borel 1904, p. 516).

Este tipo de objeciones valdrán, según Borel, para todo razonamiento en el cual se suponga hecha una elección arbitraria una cantidad infinita no numerable de veces pues tales razonamientos están fuera del dominio de las matemáticas. Sin embargo, la cuestión que quedó abierta después de estas objeciones fue la posibilidad de hacer una elección arbitraria una cantidad numerable de veces (lo que a veces es llamado el Axioma de Elección Numerable).

Después de la publicación de este texto de Borel, Jacques Hadamard publicó, en 1905, Cinco cartas sobre la teoría de conjuntos, ${ }^{21}$ que son cartas escritas tanto por el propio Hadamard como por René Baire, Borel y Henri Lebesgue. En ocasiones se habla de estos tres matemáticos franceses (Baire, Borel y Lebesgue) refiriéndose a ellos como semiintuicionistas o como los empiristas franceses y su posición frente al Axioma de Elección juega un papel importante en esta denominación.

La primera de las cinco cartas es de Hadamard a Borel. En ella Hadamard responde al artículo de Borel publicado en los Mathematische Annalen (Borel 1904) y expresa su desacuerdo con él. La primera objeción que encuentra Hadamard al razonamiento que hace Borel en su artículo (sobre un número infinito de elecciones sucesivas y por tanto dependientes unas de otras) es que en la prueba de Zermelo las elecciones son independientes, no se hacen de manera sucesiva. Hadamard argumenta también que para él no hay diferencia entre una cantidad no numerable de elecciones y una cantidad numerable nuevamente debido a la independencia de las mismas.

Con respecto a la manera de llevar a cabo las elecciones, Hadamard se pregunta si existiría alguna manera efectiva de que alguien las hiciera y dice que Zermelo no da ningún método para hacerlo y que parecería poco probable que alguien lo pudiera hacer. Lo que Zermelo había hecho era enunciar su existencia. La dife-

\footnotetext{
${ }^{21}$ La publicación de estas cartas fue una decisión tomada por sus cuatro autores al reunirse en una sesión de la Sociedad Matemática de Francia, el 4 de mayo de 1905. Allí Borel y Lebesgue hablaron sobre los principios de la teoría de conjuntos.
} 
rencia esencial, que Hadamard atribuye a Jules Tannery (Tannery 1897, pp. 132 139), radica entre i) establecer que una función existe y ii) que puede ser especificada de manera única. Tannery, en el caso de i), dice que la función puede ser definida y, en el caso de ii), que la función puede ser descrita. ${ }^{22}$

Hadamard dice que muchos problemas matemáticos tendrían un significado completamente distinto (y soluciones distintas) si la condición i) fuera reemplazada por la condición ii). Además, afirma que el propio Borel había utilizado, sobre todo en algunos teoremas sobre la convergencia de series complejas, funciones cuya existencia se podía probar pero que no podían ser definidas de manera única.

Tú empleas correspondencias cuya existencia constatas sin poder, no obstante, describirlas; en tu importante razonamiento sobre series que admiten a su círculo de convergencia como cortadura: si uno se limitara a series enteras cuya ley de formación puede ser descrita, la opinión antigua (a saber, que las series enteras que admiten a su círculo de convergencia como cortadura son la excepción) debería ser considerada como la verdadera. (Hadamard 1905, p. 262)

Finalmente, Hadamard argumenta que la existencia de la función de elección en el artículo de Zermelo es equivalente a la posibilidad de escoger un elemento de cualquier conjunto dado. ${ }^{23}$

Borel envió la carta de Hadamard a René Baire, quien respondió al propio Hadamard. Es justo decir que Baire estaba de acuerdo con Borel pero que su posición era más radical. Baire dice que constantemente se habla de conjuntos dados y afirma que esta expresión no siempre tiene sentido: "En particular, cuando se dice que un conjunto está dado [...] es falso para mí considerar a las partes de este conjunto como dadas" (Hadamard 1905, p. 264). Es decir, desde el punto de vista de Baire, es falso concebir a los subconjuntos de un conjunto dado como dados. Una vez establecido este punto de vista resulta evidente que, para este autor, es fácil rechazar la idea de que se pueda llevar a cabo una elección en cada uno de estos subconjuntos.

Baire argumenta de esta forma que lo que Zermelo hizo en su artículo fue mostrar que "no percibimos una contradicción al suponer que en cada conjunto que está definido, los elementos guardan entre sí relaciones de posición idénticas a las que los elementos de un conjunto bien ordenado tienen. Para decir entonces que uno ha establecido que todo conjunto puede ser llevado a la forma de un conjunto bien ordenado es necesario dar a estas palabras una extensión extraordinaria, y tramposa, debo agregar" (Hadamard 1905, p. 264).

En otras palabras, el resultado de Zermelo es consistente pero carece de sentido. Finalmente, Baire termina su carta diciendo que todo en matemáticas debería ser reducido a lo finito.

\footnotetext{
22 Estos términos han cambiado; en la actualidad si se cumple ii) se dice que la función está definida.

${ }^{23}$ Es importante notar que esto en realidad es falso; sin embargo, esta confusión vuelve a aparecer muchos años después en los trabajos de Arnaud Denjoy (1946 p. 111) y Paul Lévy (1950, p. 23).
} 
Poco tiempo después Borel pidió la opinión de Lebesgue sobre el mismo tema. Lebesgue le contesta con una carta larga en la cual toma una posición similar a la de Borel y menos radical que la de Baire. Lebesgue retoma la nomenclatura dada por Borel sobre los problemas A y B (que enunciamos líneas arriba) y dice que él duda fuertemente que se pueda dar una solución general al problema $B$, sobre todo si se acepta la noción de Cantor de que definir un conjunto $M$ es nombrar una propiedad $P$ que pertenece a ciertos elementos de un conjunto $\mathrm{N}$ previamente definido y que caracterice por definición a los elementos de M. ${ }^{24}$

Después de esto, Lebesgue dice que el problema central es el siguiente: ¿Se puede demostrar la existencia de un objeto matemático sin definirlo? (Hadamard 1905, p. 265). La respuesta a esta cuestión evidentemente no es sencilla. Lebesgue juzga que es un problema de convención y dice que él cree que no se puede construir sólidamente en matemáticas sino al aceptar que solo se puede demostrar la existencia de un objeto al definirlo (de manera explícita y única). ${ }^{25}$

En un artículo escrito también en 1905, pero no publicado hasta 1971 (Lebesgue 1971), Lebesgue retoma este punto y considera que la pregunta fundamental debe ser qué funciones se pueden introducir legítimamente. Argumenta que la definición de función usual es demasiado vaga pues no indica por qué medios se puede nombrar o especificar una función y arguye, con un intuicionismo comparable al de Brouwer, que aun para la función que vale 0 en todos los racionales y 1 en los irracionales (que se puede definir analíticamente) no se puede calcular el valor para algunos valores de la variable como, por ejemplo, la constante de Euler. ${ }^{26}$

En este artículo Lebesgue hace alusión a una división hecha por Paul du Bois Reymond (1887) de la comunidad matemática: los idealistas y los empiristas. ${ }^{27}$ Los empiristas solo aceptan las funciones que pueden ser definidas mientras que los idealistas aceptan otras también. ${ }^{28} \mathrm{Y}$ es esta distinción la que Lebesgue convirtió en la posición clave que definiría su filosofía empírica de las matemáticas.

No obstante esta posición tomada por Lebesgue es importante notar (como él mismo lo hace en su carta) que en su tesis doctoral él demuestra la existencia

\footnotetext{
${ }^{24}$ Es importante aclarar que, aun cuando Lebesgue explícitamente le atribuye esta definición (basada en lo que hoy llamaríamos el axioma de separación) a Cantor, en realidad no coincide con la de Cantor. La noción de conjunto presentada por Cantor (1883) podría justificar parcialmente la frase de Lebesgue pues ahí Cantor dice que un conjunto puede ser visto como una totalidad unida por una ley; sin embargo, la definición de conjunto presente en Cantor (1895) no la justifica pues ahí Cantor define a un conjunto como cualquier colección de objetos de nuestra intuición o pensamiento.

${ }^{25}$ Este punto de vista contrasta con el expresado por Hilbert sobre la existencia en matemáticas, que señalamos en la primera parte de este artículo.

${ }^{26}$ La constante de Euler se denota usualmente por $\gamma$ y su valor es $\lim _{n \rightarrow \infty}\left(\sum_{k=1}^{n} \frac{1}{k}-\ln n\right)$. La pregunta de
si es un número racional o irracional permanece abierta.

${ }^{27}$ Estos términos han cambiado su sentido a lo largo de los años, según Moore (1982) en un lenguaje filosófico contemporáneo los idealistas de Lebesgue serían llamados realistas y los empiristas serían llamados idealistas.

${ }^{28}$ Según este punto de vista, Zermelo y Hadamard serían idealistas mientras que Lebesgue, Baire y Borel serían empiristas.
} 
de conjuntos L-medibles que no son B-medibles pero que le parece dudoso que alguien pudiera jamás nombrar uno. ${ }^{29}$

Ahora bien, además de considerarse empirista, Lebesgue define su posición en torno de la existencia en matemáticas como constructivista y se considera en este punto cercano a Kronecker y Drach. Para nosotros es claro que son precisamente estas posiciones las que no le permiten aceptar la demostración de Zermelo. Para Lebesgue no había manera de garantizar que los elementos distinguidos en la prueba de Zermelo permanecieran invariantes a lo largo de la demostración dado lo arbitrario de la elección en la que no se precisaba una manera de determinarlos.

Borel envió las cartas de Baire y Lebesgue a Hadamard, quien seguía sosteniendo su punto de vista original y afirmaba también que es posible mostrar la existencia de un objeto sin definirlo. La manera en que se prueba la existencia de un objeto carece de importancia, según Hadamard.

Hadamard argumentó que la manera en la que el problema había sido interpretado por Baire, Borel y Lebesgue era muy distinta a la suya (y a la de Zermelo). Hadamard dice que para sus tres oponentes la pregunta en cuestión era ¿podemos bien ordenar un conjunto?, cuando para él la pregunta es ¿es posible un buen orden? Esta diferencia en la interpretación del problema que aprecia Hadamard lo lleva a concluir que Baire, Borel y Lebesgue ven las cosas desde un punto de vista completamente subjetivo, psicológico y, por tanto, contrario a la naturaleza de las matemáticas.

Finalmente, en la última de estas cartas, escrita por Borel a Hadamard, este confirma que entre los cuatro autores se sostienen puntos de vista distintos. Vale la pena notar que en esta carta Borel cita un argumento utilizado por él mismo en su libro Leçons sur les fonctions de variables réelles (Borel 1905), donde afirma haber definido una función real que no estuviera en la clase de Baire $0,1, \ldots, n$ para cualquier $n$ finita dada. En 1918 Sierpinski (Sierpinski 1918) ${ }^{30}$ señaló que para ello Borel había hecho uso del axioma de elección y mostró un vínculo entre estos resultados y la posibilidad de determinar un conjunto no L-medible. De esta manera, la discusión llevada a cabo entre estos matemáticos a propósito del axioma de elección desemboca en el problema de la relación de dicho axioma con la teoría de la medida. En las próximas dos secciones nos ocuparemos de la teoría de la medida y de su relación con el axioma de elección.

\section{La teoría de la medida: Jordan, Borel y Lebesgue}

El concepto matemático de medida surgió hacia finales del siglo XIX vinculado primordial, pero no exclusivamente, con la teoría de la integración. Durante las dos últimas décadas de ese siglo la teoría de la integración se enfocó particular-

\footnotetext{
${ }^{29}$ Estudiaremos con detalle más adelante, en la sección dedicada al problema de la medida, el argumento proporcionado por Lebesgue.

${ }^{30}$ Las citas de este artículo están tomadas de la versión que aparece en la obras de Sierpinski (1974).
} 
mente en algunas propiedades de los conjuntos infinitos. En particular el descubrimiento de la existencia de algunos conjuntos densos en ninguna parte con contenido exterior positivo ${ }^{31}$ mostró la relevancia que las propiedades relacionadas con la medida de conjuntos podían tener para la teoría de la integración y con rapidez esto llevó a la introducción de distintas teorías de la medida. ${ }^{32}$

En 1892, Camille Jordan publicó un artículo llamado "Remarques sur les intégrales définies". En este texto Jordan dice que el papel que la función juega en la integral definida ha sido ya bien comprendido y, por tanto, se propone estudiar la influencia que el dominio de la función tiene sobre esta integral.

Lo primero que Jordan (1982) muestra es que a un conjunto cualquiera E corresponden dos números determinados E’ y E”, que llama su extensión ${ }^{33}$ interior y su extensión exterior. Si estos dos números coinciden se dirá que el conjunto E es medible y que tiene por extensión al número E' = E”. Un hecho que es importante notar es que la extensión de un conjunto será una longitud, un área, un volumen, etc. dependiendo de la dimensión del conjunto, es decir el concepto de extensión que Jordan define será de antemano una generalización de los conceptos de longitud, área, etc. ya existentes.

Como veremos más adelante, otro punto importante de este proceso llevado a cabo por Jordan es que es constructivo; se puede resumir de la siguiente manera:

Sea E un conjunto en el plano y consideremos una descomposición de este en cuadrados cuyos lados son paralelos a los ejes usuales y tienen longitud r. El conjunto de cuadrados cuyos puntos son todos interiores a E forma un dominio $\mathrm{S}$ en el interior ${ }^{34}$ de E. El conjunto de cuadrados que son interiores a $\mathrm{E}$ o contienen un punto en su frontera forman un nuevo dominio $S+S^{\prime}$ que contiene a $E$. Como S y S + S' están formados a partir de cuadrados, sus áreas están determinadas; llamémoslas $\mathrm{S}$ y $\mathrm{S}+\mathrm{S}$ '. Ahora, si r tiende a $\mathrm{O}, \mathrm{S}$ y $\mathrm{S}+\mathrm{S}$ ' tenderán hacia límites fijos, $a$ y A respectivamente y si $a=\mathrm{A}$, entonces diremos que $\mathrm{E}$ es medible.

Jordan hace ver que este proceso es independiente de la descomposición del plano y por tanto tiene sentido llamar a $a=\mathrm{e}_{i}$ la extensión interior y a $A=\mathrm{e}_{e}$ la extensión exterior del conjunto E.

Una consecuencia importante de la definición de conjunto medible que da Jordan es que si $E=\bigcup_{i=1}^{n} E_{i}$ donde los conjuntos $E_{i}$ son disjuntos, entonces $\sum_{i=1}^{n} e_{i}\left(E_{i}\right) \leq e_{i}(E) \leq e_{e}(E) \leq \sum_{i=1}^{n} e_{e}\left(E_{i}\right)$ y por tanto, si los $E_{i}$ son medibles $E$ también lo es. Es importante notar, sobre todo con relación a lo que mostraremos a continuación, que esta propiedad aditiva que cumplen los conjuntos medibles es una propiedad finita, es decir si el conjunto E se descompusiera en una cantidad numerable de conjuntos no se tendría una desigualdad equivalente.

\footnotetext{
${ }^{31}$ Se dice que un conjunto es denso en ninguna parte si la cerradura de su interior es vacía.

${ }^{32}$ Una historia de la teoría de la medida se puede encontrar en Hawkins (1975). Es importante señalar que este artículo no pretende estudiar los orígenes de esta teoría y por tanto en él no se mencionan a autores como Peano o el propio Cantor en relación con sus trabajos en esta rama.

${ }^{33}$ Actualmente se usa la palabra contenido en lugar de extensión; la palabra utilizada por Jordan es étendue.

${ }^{34}$ Jordan proporciona las definiciones de todos los conceptos topológicos que utiliza en el mismo artículo.
} 
Esta teoría de la medida definida por Jordan contrasta fuertemente con la teoría de la medida que desarrollará Émile Borel. El primer punto de contraste proviene del hecho de que el interés primario de Borel por la teoría de la medida es muy distinto al de Jordan; su motivación principal yace en la teoría de funciones de variable compleja.

Para definir su medida Borel considera únicamente conjuntos acotados en el intervalo $[0,1]$ y hace lo siguiente:

(i) Si un conjunto se forma a partir de todos los puntos contenidos en una infinidad numerable de intervalos ajenos entre sí con longitud total S, se dirá que el conjunto tiene medida S. Es decir, si se tiene una familia numerable de intervalos disjuntos $\left\{\mathrm{I}_{n}\right\}$ contenidos en $[0,1]$, entonces la medida del conjunto $\bigcup_{n} I_{n}$ será $m\left(\bigcup_{n}^{n} I_{n}\right)=\sum_{n} m\left(I_{n}\right)$ donde $m\left(I_{n}\right)$ se define como la longitud usual del intervalo.

(ii) Si dos conjuntos disjuntos tienen medidas $\mathrm{S}$ y S' su unión tiene medida $S+$ S'. En general, si se tiene una cantidad numerable de conjuntos disjuntos de medidas $s_{1}, s_{2}, \ldots, s_{n}, \ldots$, su suma tiene medida $s_{1}+s_{2}+\ldots+$ $s_{n}+\ldots$ En otras palabras, si se tiene una familia numerable de conjuntos disjuntos $\left\{E_{n}\right\}$ en $[0,1]$, entonces la medida del conjunto $\bigcup_{n} E_{n}$ será $m\left(\bigcup_{n} E_{n}\right)=\sum_{n} m\left(E_{n}\right)$.

(iii) $\mathrm{Si} \mathrm{E}_{1} \subseteq \mathrm{E}_{2}$ son dos conjuntos contenidos en $[0,1]$ cuyas medidas han sido dadas, entonces el conjunto $E_{2}-E_{1}$ tendrá medida $m\left(E_{2}\right)-m\left(E_{1}\right)$.

Los conjuntos cuya medida se puede determinar en virtud de las definiciones precedentes serán llamados por Borel conjuntos medibles, sin implicar con esto que no sea posible dar una definición de la medida de otros conjuntos. ${ }^{35}$

Las tres propiedades con las que Borel define su medida también sirven para definir las dos operaciones con las cuales es posible construir conjuntos medibles; la única restricción que impone Borel (de manera implícita en su procedimiento) es que las operaciones se apliquen únicamente una cantidad numerable de veces. Esto tiene como consecuencia que la cardinalidad de la clase de los conjuntos medibles es igual a la del continuo. ${ }^{36}$

Ahora bien, Borel argumenta a favor de la medida que ha definido (versus una medida que se defina sobre una clase de conjuntos más grande) diciendo que es crucial para él que tenga las propiedades fundamentales que ha dado. En otras palabras, es esencial darse cuenta de que una definición de medida no puede ser útil si no posee ciertas propiedades; de manera que estas propiedades en este caso han sido dadas a priori y es a partir de estas que la clase de conjuntos medibles se obtiene. Esta presentación contrasta con la de Jordan pues este

\footnotetext{
${ }^{35}$ Como resultado de esta definición de medida Borel obtiene que la medida de un conjunto nunca será una cantidad negativa, que un conjunto puede tener medida cero (aunque tenga la potencia del continuo) y que un conjunto numerable siempre tiene medida cero.

${ }^{36}$ Lebesgue prueba esto en 1902 en su tesis doctoral (Lebesgue 1902, p. 212) y hacemos notar que depende del axioma de elección.
} 
método de proceder no ha sido generativo sino descriptivo. Borel dice que "esta manera de proceder presenta grandes analogías con los métodos introducidos por Jules Drach en Algebra y en la teoría de ecuaciones diferenciales [...]. En todos los casos se procede con la misma idea fundamental: definir los elementos nuevos que se introducen con ayuda de sus propiedades esenciales, es decir, de aquellas que son estrictamente indispensables para los razonamientos que deben seguir" (Borel 1898, p. 48).

Esta teoría, junto con la creada por Jordan, jugaría un papel importante en el desarrollo de la teoría de la medida de Lebesgue.

En 1902 se publicó la tesis doctoral de Lebesgue "Intégrale, Longueur, Aire" (Lebesgue 1902). ${ }^{37} \mathrm{Su}$ objetivo es el de dar definiciones precisas a ciertos conceptos que aparecen en el Análisis, en particular, la integral definida, la longitud de una curva y el área de una superficie. Esto implica que el interés de Lebesgue al desarrollar la teoría de la medida que necesita para estos objetivos coincide con el de Jordan, es decir, radica en la teoría de la integración, y por tanto difiere del de Borel.

Lo primero que tiene que hacer Lebesgue para lograr estos objetivos es introducir una teoría de la medida que resultará distinta tanto de la de Jordan como de la de Borel. Para hacer esto enuncia el problema de la medida:

Nos proponemos asignar a cada conjunto acotado un número positivo o nulo que llamaremos su medida y que satisfará las siguientes condiciones:

1. Existen conjuntos cuya medida no es nula.

2. Dos conjuntos iguales ${ }^{38}$ tienen la misma medida.

3. La medida de la suma de un número finito o de una infinidad numerable de conjuntos ajenos entre sí es la suma de las medidas de estos conjuntos.

No resolveremos este problema de la medida sino para los conjuntos que llamaremos medibles. (Lebesgue 1972, vol. 1, p. 208)

Estas tres condiciones se pueden concebir como los tres axiomas (o propiedades esenciales en el lenguaje de Borel) que debe satisfacer una medida para llevar a cabo los objetivos de Lebesgue. El procedimiento que este último llevará a cabo comparte con el de Borel la manera en la que ha sido planteado pero comparte con el proceso llevado a cabo por Jordan el hecho de que la medida que debe satisfacer el problema dado será obtenida a través de un proceso explicitado.

Ahora bien, para resolver este problema de la medida, Lebesgue resuelve primero el problema de la unicidad, es decir la posibilidad de que el problema planteado tenga más de una solución. Lebesgue hace notar que, si el problema de la medida planteado tiene solución, tendrá claramente más de una, ya que cualquier múltiplo de la solución dada también será solución. Para corregir esto se puede escoger arbitrariamente la medida de un segmento y fijar como 1 y si este segmento es tomado como unidad, la medida de cada intervalo acotado será su longitud en el sentido usual.

\footnotetext{
${ }^{37}$ Las citas se tomaron de Lebesgue (1972).

${ }^{38} \mathrm{La}$ igualdad entre conjuntos aquí se refiere a la invariancia bajo traslaciones, es decir, congruencia.
} 
Esto ciertamente resuelve el problema de la unicidad que Lebesgue plantea, pero vale la pena notar que no resuelve el problema de la unicidad en un sentido general, que no será atacado por Lebesgue. ${ }^{39}$

Lebesgue observa que como resultado de las condiciones del problema de la medida se tendrá que los conjuntos que contengan un solo punto tendrán medida cero (ya que los conjuntos acotados con un número infinito de puntos deben tener medida finita) y que la medida de intervalos acotados es positiva.

El proceso llevado a cabo por Lebesgue para construir su medida se puede resumir de la siguiente manera: Sea $\mathrm{E}$ un conjunto acotado. Es posible encerrar a los puntos de $\mathrm{E}$ en una colección finita o numerable de intervalos de una infinidad de maneras. Sea $\mathrm{E}_{1}$ el conjunto de puntos de una de estas colecciones de intervalos. La medida $m(\mathrm{E})$ de $\mathrm{E}$ es a lo más igual a la medida de $\mathrm{E}_{1}$. El ínfimo sobre todas las posibles colecciones $\mathrm{E}_{1}$ es una cota superior para $m(\mathrm{E})$ y se define a la medida exterior de $E, m_{e}(E)$, como este ínfimo. Es decir,

$$
m_{e}(E)=\left\{\inf \left(m\left(E_{1}\right)\right): E_{1}=\bigcup_{n} I_{n} \supseteq E y I_{n} \cap I_{m}=\varnothing \sin \neq m\right\}
$$

donde $\mathrm{I}_{n}$ denota un intervalo.

Ahora, si todos los puntos de $E$ pertenecen a un segmento fijo $A B$, la medida de $A B-E$ es a lo más $m_{e}(A B-E)$ y por tanto la medida de $E$ es por lo menos $m(\mathrm{AB})-m_{e}(\mathrm{AB}-\mathrm{E})$. Este número es la medida interior, $m_{i}(\mathrm{E})$, de $\mathrm{E}$ y es independiente del segmento $\mathrm{AB}$. Es fácil ver que $m_{e}(\mathrm{E})^{3} m_{i}(\mathrm{E})$. Lebesgue llamará conjuntos medibles a aquellos conjuntos cuya medida exterior e interior sean iguales; el valor común de estos dos números será la medida del conjunto.

Se prueba que esta medida cumple que si $\mathrm{E}_{1}, \mathrm{E}_{2}, \ldots$ son una infinidad numerable de conjuntos medibles, entonces tanto $\bigcup_{n=1}^{\infty} E_{n}$ como $\bigcap_{n=1}^{\infty} E_{n}$ son medibles. Nuevamente esto resulta de gran importancia pues es a través de estas dos operaciones aplicadas un número numerable de veces que se pueden obtener nuevos conjuntos medibles. La diferencia con la medida de Borel es que Lebesgue no parte únicamente de intervalos y, por tanto, la clase de conjuntos Lebesgue medibles es más grande que la de los conjuntos Borel medibles. De hecho, es fácil ver que los conjuntos medibles en el sentido de Borel (los conjuntos que Lebesgue llama B-medibles) son medibles para Lebesgue pero no viceversa.

[Los conjuntos B-medibles] están definidos por una infinidad numerable de condiciones, su conjunto tiene la potencia del continuo [...]. El conjunto E formado por los puntos con abscisas $x=\frac{a_{1}}{3}+\frac{a_{2}}{3^{2}}+\frac{a_{3}}{3^{3}}+\ldots$ donde las $a_{i}$ son iguales a 0 o 2 siendo perfecto es B-medible. Su complemento está formado por un intervalo $\left(\frac{1}{3}, \frac{2}{3}\right)$ de longitud $\frac{1}{3}$, de dos intervalos $\left(\frac{1}{9}, \frac{2}{9}\right)$,

\footnotetext{
${ }^{39}$ El problema de la unicidad en un sentido general plantea la posibilidad de la existencia de dos medidas que satisfagan las propiedades 1-3 dadas por Lebesgue pero que no coincidan en todos los conjuntos, es decir, que como funciones sean distintas. En efecto, quien ataca este problema es Banach (1923), veinte años después.
} 
$\left(\frac{2}{3}+\frac{1}{9}, \frac{2}{3}+\frac{2}{9}\right)$ de longitud $\frac{1}{3^{2}}$, de cuatro intervalos de longitud $\frac{1}{3^{3}}$, etc., por tanto tiene medida $\frac{1}{3}+2 \frac{1}{3^{2}}+2^{2} \frac{1}{3^{3}}+\ldots=1$ y por consiguiente $E$ es de medida nula. E tiene la potencia del continuo, por tanto se puede formar con los puntos de $\mathrm{E}$ una infinidad de conjuntos que, teniendo medida exterior nula, sean medibles. La potencia del conjunto de estos conjuntos es aquella del conjunto de conjuntos de puntos; por tanto existen conjuntos medibles que no son B-medibles, y la potencia del conjunto de conjuntos medibles es aquella del conjunto de conjuntos de puntos. (Lebesgue 1972, vol. 1, pp. 212-213)

Por otro lado, la diferencia entre las medidas de Jordan y Lebesgue radica en los puntos interiores. Si un conjunto tiene puntos interiores, entonces las medidas coinciden, pero si este no es el caso entonces el conjunto no será medible en el sentido de Jordan. De esta manera, el conjunto de conjuntos Jordan medibles (J-medibles) también es un subconjunto propio de la clase de conjuntos Lebesgue medibles, pero en este caso se cumple que "el conjunto de conjuntos J-medibles tiene la misma potencia que el conjunto de conjuntos de puntos” (Lebesgue 1972, p. 214).

Estos resultados sobre las cardinalidades de los conjuntos B-medibles, J-medibles y L-medibles llevan fácilmente al lector a preguntarse si el conjunto de conjuntos L-medibles coincide con el conjunto de todos los conjuntos acotados, es decir, si cualquier conjunto acotado en la recta es L-medible. Lebesgue no aborda este tema en lo absoluto; su trato con este problema se limita a la siguiente frase: "No se ha demostrado que el problema de la medida sea imposible para los conjuntos (si es que existen) cuyas medidas interiores y exteriores sean desiguales. Pero en lo que sigue solo encontraremos conjuntos medibles" (Lebesgue 1972, vol. 1, p. 211). De esta manera, aunque Lebesgue únicamente tratará con conjuntos medibles, la posible existencia de un conjunto acotado no medible queda abierta y es en respuesta a esta cuestión que se obtiene un substancial vínculo entre esta teoría y el axioma de elección.

\section{La teoría de la medida y el axioma de elección}

En 1905, G. Vitali publicó el artículo Sul problema della misura dei gruppi di punti di una retta, en el cual muestra que existe un conjunto que no es susceptible de ser medido con la medida de Lebesgue. Para hacer esto define la siguiente relación de equivalencia en R: si $x$ y y son números reales, entonces $x \sim y$ si $x-y$ es racional. Esta relación de equivalencia divide a $\mathrm{R}$ en clases disjuntas y se considera el conjunto $\mathrm{V}$ formado con un representante de cada clase. Se prueba fácilmente que $\mathrm{R}=\bigcup_{i=1}^{\infty}\left(q_{i} \oplus \mathrm{V}\right)$ donde $\left(q_{i}\right)$ es una enumeración del conjunto de los números racionales. A partir de esto, si se supone que $m(\mathrm{~V})>0$ Vitali muestra que se llega a una contradicción y si $m(V)=0$ entonces se sigue que $m(R)=0$ y por tanto se concluye que $\mathrm{V}$ no es L-medible.

La demostración de Vitali es concisa y muy clara; sin embargo, no fue poca la controversia que causó puesto que para obtener al conjunto $\mathrm{V}$ es claramente nece- 
sario utilizar el axioma de elección. Es decir, lo que Vitali había mostrado es que, si se permite el uso de este axioma en el análisis matemático, entonces el problema de la medida de Lebesgue, como él lo plantea en su tesis, no tiene solución, es decir que no existe una medida real, no negativa, definida sobre todos los conjuntos acotados, que sea invariante bajo traslaciones, numerablemente aditiva y tal que el intervalo unitario tenga medida $14^{40} \mathrm{O}$ en palabras de Vitali: "el problema de la medida de grupos de puntos de una recta es imposible [...]. Nuestro resultado significa que la posibilidad del problema de la medida de grupos de puntos de una recta y aquella de bien ordenar el continuo no pueden coexistir" (Vitali 1905, p.5).

Esta demostración de Vitali nos remite nuevamente al artículo de Sierpinski mencionado al final de la sección III, (Sierpinski 1918) pues, como ya habíamos destacado, Sierpinski señala un uso tácito por parte de Borel del axioma pero además muestra que si cualquier función $f(x)$ de la clase 2 de Baire se pudiera representar de la forma $\sum_{i=1}^{\infty} \sum_{j=1}^{\infty} P_{i, j}(x)$ donde $P_{i, j}(x)$ es un polinomio real para cada $i$ y $j$ (como lo asume Borel) entonces se podría determinar explícitamente un ejemplo de un conjunto no L-medible.

Sierpinski en ese mismo artículo dedica la sección 5 a la relación entre el axioma de Zermelo y la teoría de la medida.

Sin basarnos sobre el axioma del Sr. Zermelo no sabemos demostrar el teorema fundamental de la teoría de la medida lebesguiana, teorema a partir del cual el conjunto unión de una infinidad numerable de conjuntos medibles es un conjunto medible [...]. Tampoco sabemos demostrar, si no apelamos al axioma del Sr. Zermelo, que el conjunto unión de una infinidad numerable de conjuntos medibles $E_{1}, E_{2}, E_{3}, \ldots$ que no tienen puntos en común dos a dos es un conjunto medible, y su medida es igual a la suma de las medidas de los conjuntos $\mathrm{E}_{1}, \mathrm{E}_{2}, \mathrm{E}_{3}, \ldots$ En particular no sabemos demostrar, sin invocar este axioma, que el conjunto unión de una infinidad numerable de conjuntos de medida nula no puede tener una medida positiva. Notemos que si supiéramos demostrar esta última proposición sin basarnos sobre el axioma del Sr. Zermelo, podríamos demostrar sin este axioma que el continuo no puede descomponerse en una infinidad numerable de conjuntos numerables puesto que se puede demostrar sin recurrir al axioma citado que todo conjunto numerable es de medida nula. (Sierpinski 1974, Vol. 2, p. 233)

Y concluye esta sección diciendo que "no se puede probar, sin ayuda del axioma del Sr. Zermelo que existen conjuntos no medibles en el sentido del Sr. Lebesgue" (Sierpinski 1974, p. 234). Sierpinski agrega como nota al pie que:

En su memoria C. Burstin "Eigenschaften meßbarer und nichtmeßbarer Mengen" (Sitzungsberichte der Kaiserlichen Akademie der Wissenschaften in Wien. In Sitzungsberichte der Mathematisch-Naturwissenschaftlichen Klasse 123, Abteilungn IIa 1914, pp. 1525-1551) que además contiene varios teoremas erróneos, el Sr. C. Burstin anuncia que ha demostrado, sin la ayuda del axioma de elección, la existencia de conjuntos no medibles (p. 1549). En realidad la demostración que da el Sr. Burstin hace alusiones varias veces al axioma del Sr. Zermelo. (Sierpinski 1974, p. 234).

${ }^{40}$ En esta perspectiva es importante hacer notar que en 1970 Solovay demostró que el axioma de elección es necesario para demostrar la existencia de un conjunto no Lebesgue medible. 
Las secciones 7 y 8 del mismo artículo tratan el tema de conjuntos que no son Lmedibles. La sección 7 se intitula "El axioma del Sr. Zermelo en la construcción de ejemplos" y comienza así:

Se han construido muchos ejemplos de conjuntos o funciones que tengan propiedades dadas con la ayuda del axioma del Sr. Zermelo. Tales son todos los ejemplos conocidos de conjuntos no medibles (ejemplos de Vitali, Lebesgue, Van Vleck, Hausdorff); el ejemplo de un conjunto de puntos de potencia $\aleph_{1}$ (Hardy); el ejemplo de un conjunto no numerable de puntos que no contiene ningún subconjunto perfecto (F. Bernstein); el ejemplo de un conjunto de puntos que al igual que su complemento es de la segunda categoría ${ }^{41}$ sobre cada segmento (Mahlo); el ejemplo de un conjunto no numerable de puntos que es de la primera categoría sobre todo conjunto perfecto ( $\mathrm{Lu}$ sin); el ejemplo de una función discontinua que satisfaga la ecuación funcional $f(x+y)=f(x)+f(y)$ (Hamel); el ejemplo de un conjunto plano tal que toda recta lo interseca en dos puntos solamente (Mazurkiewicz). No sabemos demostrar la existencia de ninguno de estos ejemplos sin apoyarnos sobre el axioma del Sr. Zermelo. (Sierpinski 1974, p. 244)

Finalmente, Sierpinski termina su artículo con el siguiente comentario (que evidentemente es posible solo por el Axioma de Zermelo) que caracteriza de una manera muy sugestiva a los conjuntos no medibles:

[S]i uno divide en clases a todos los conjuntos medibles acomodando en la misma clase dos conjuntos si y solo si difieren entre sí por un conjunto de medida nula, el conjunto de todas las clases diferentes tiene la potencia del continuo. Esta proposición se sigue fácilmente del teorema siguiente: todo conjunto medible difiere en a lo más un conjunto de medida nula de la unión de una serie numerable de conjuntos cerrados. No obstante que el conjunto de todos los conjuntos no medibles tenga la misma potencia que el conjunto de todos los conjuntos medibles, la diversidad de los conjuntos no medibles es así infinitamente más grande que la de los conjuntos medibles. (Sierpinski 1974, p. 255)

Es interesante comparar este punto de vista con el que Banach expresará en 1929 sobre los conjuntos no medibles, como veremos más adelante; sin embargo, también resulta relevante estudiar una variación del problema de la medida planteada por Hausdorff en 1914.

Hausdorff muestra que el problema de la medida planteado por Lebesgue no tiene solución en el espacio $n$-dimensional ( $\operatorname{con} n^{3} 3$ ) construyendo conjuntos del tipo de Vitali (Hausdorff 1914). Para hacer esto se plantea la siguiente pregunta, que se conoce como el problema amplio de la medida: ¿Es posible asignar a cada conjunto acotado $\mathrm{E}$ de un espacio de $n$ dimensiones un número $m(\mathrm{E})$ que satisfaga las condiciones siguientes?

(i) $m(\mathrm{E}) \geq 0$

(ii) $m\left(\mathrm{E}_{0}\right)=1$ para algún conjunto $\mathrm{E}_{0}$ del espacio considerado

\footnotetext{
${ }^{41}$ Se dice que un conjunto es de la primera categoría si es la unión numerable de conjuntos densos en ninguna parte y se dice que un conjunto es de la segunda categoría si no es de la primera categoría.
} 
(iii) $m\left(\mathrm{E}_{1} \cup \mathrm{E}_{2}\right)=m\left(\mathrm{E}_{1}\right)+m\left(\mathrm{E}_{2}\right)$ si $\mathrm{E}_{1} \mathrm{y} \mathrm{E}_{2}$ son disjuntos

(iv) $m\left(E_{1}\right)=m\left(E_{2}\right)$ si los conjuntos $E_{1} y E_{2}$ son superponibles

La principal diferencia con el problema de la medida planteado por Lebesgue radica en la condición 3, puesto que Hausdorff únicamente impone una condición de aditividad finita mientras que para Lebesgue la aditividad debía ser numerable.

Lo que Hausdorff muestra es que este problema es imposible para el espacio de tres o más dimensiones. Demuestra (con ayuda del axioma de elección) que existe una descomposición de la superficie (que se puede suponer igual a 1) de una esfera (en el espacio tridimensional y por ende en cualquier espacio de dimensión mayor) en cuatro conjuntos, $\mathrm{A}, \mathrm{B}, \mathrm{C}$ y $\mathrm{Q}$ tales que $\mathrm{Q}$ es numerable, $\mathrm{A} \cong \mathrm{B} \cong \mathrm{C}$ y $\mathrm{A} \cong \mathrm{B} \cup \mathrm{C}$. Por tanto, si existiera una medida que satisfaga las condiciones (i)-(iv), se tendría que la medida de $Q$ es nula y que $m(A)=m(B)=m(c)=\frac{1}{3}$ y $m(A)=m(B \cup C)=\frac{1}{2}$ lo cual es evidentemente una contradicción.

En 1923 Banach publica el artículo "Sur le problème de la mesure" (Banach 1923) y demuestra que en los espacios de dimensión uno y dos el problema amplio de la medida planteado por Hausdorff se resuelve afirmativamente y para hacer esto también recurre al axioma de elección. A partir de la publicación de este artículo se ve ya un claro y estrecho vínculo entre este axioma y el problema de la medida.

En el mismo artículo Banach presenta un problema que le ha sido planteado por Ruziewicz y que es el siguiente: ¿Existe una operación $f(\mathrm{X})$ que satisfaga las condiciones siguientes?

(i) $f(X)$ está definida para todo conjunto L-medible de un espacio de $n$ dimensiones

(ii) $f(X) \geq 0$

(iii) $f\left(X_{0}\right)=1$ para algún conjunto con medida de Lebesgue 1

(iv) $f(X \cup Y)=f(X)+f(Y)$ si $X$ y $Y$ son disjuntos

(v) $f(X)=f(Y)$ si $X \cong Y$

(vi) $f\left(X_{1}\right) \neq m\left(X_{1}\right)$ para algún conjunto $X_{1}$ que es L-medible (donde $m$ denota precisamente a la medida de Lebesgue).

Banach demuestra que el problema de Ruziewicz se resuelve afirmativamente para los espacios de una o dos dimensiones y el problema permanece abierto para los espacios de tres o más dimensiones. Esto significa que, en el caso de dimensión 1 o 2, la medida de Lebesgue no es la única medida que es finitamente aditiva, positiva, normalizada e invariante bajo traslaciones.

Un poco después, en 1929, Banach y Kuratowski publican un artículo intitulado "Sur une généralisation du problème de la mesure" (Banach \& Kuratowski 1929). En este artículo se prueba que no existe ninguna función $m(X)$ que asigne a cada conjunto $\mathrm{X} \subseteq \mathrm{E}=[0,1]$ un número real $m(\mathrm{X})$ de manera que 
(i) $m(X)=0$ si $X$ está compuesto de un solo punto;

(ii) $m\left(X_{1} \cup X_{2} \cup \ldots\right)=m\left(X_{1}\right)+m\left(X_{2}\right)+\ldots$ si los $X_{i}$ son conjuntos disjuntos $y$

(iii) $m(\mathbf{X})$ no sea idénticamente cero.

Para probar que este problema generalizado de la medida no acepta solución es necesario aceptar la hipótesis del continuo. Este último hecho junto con las demostraciones mencionadas desde 1905 llevan a los autores a concluir lo siguiente:

Como se puede ver, el hecho de que el problema de la medida no posea una solución no es de carácter geométrico (como se podría haber creído), sino que es un resultado de la Teoría de Conjuntos. En cuanto al conjunto E, ${ }^{42}$ es claro que se puede suponer que es un conjunto arbitrario de la potencia del continuo (sin ser necesariamente un intervalo). (Banach \& Kuratowski 1929, p. 130)

Ahora bien, lo que resulta sumamente interesante de esta demostración es que Banach y Kuratowski dejan de lado la condición original de Lebesgue, que pedía que los conjuntos congruentes tuvieran la misma medida. El haber introducido la noción de congruencia (o de igualdad entre conjuntos, como la llama Lebesgue) le da al problema una carga geométrica que es abandonada por los autores de este artículo. De esta manera, la generalización del problema de la medida es planteada en un marco puramente conjuntista. Es en este marco que la hipótesis del continuo juega el papel de axioma en esta prueba y se muestra que si la hipótesis del continuo fuera agregada a los axiomas usuales de la teoría de conjuntos entonces resultaría imposible construir una medida que satisficiera las condiciones establecidas por Lebesgue.

Este hecho es importante para el análisis que tratamos de presentar aquí pues, no obstante que la condición eliminada por Banach y Kuratowski fue la de medidas iguales para conjuntos iguales, la demostración presentada revela que es la condición de la aditividad numerable la que puede establecer de manera clara los vínculos entre la teoría de la medida y la hipótesis del continuo. Es la condición de aditividad numerable la que resulta incompatible con la igualdad $2^{\aleph_{0}}=\aleph_{1}$ como veremos al analizar brevemente la demostración de Banach y $\mathrm{Ku}$ ratowski.

La demostración muestra que si se puede construir una doble sucesión $\left\{A_{k}^{n}\right\}$ de subconjuntos de $\mathrm{E}$

tal que se cumplan las siguientes dos condiciones:

(i) $A_{i}^{n} \cap A_{j}^{n}=\varnothing$ para toda $n$

(ii) $E=\bigcup_{i=1}^{\infty} A_{i}^{n}$ para toda $n$,

\footnotetext{
${ }^{42}$ Se trata aquí del mismo conjunto $\mathrm{E}=[0,1]$.
} 
entonces al definir una medida en el conjunto E que satisfaga la condición de aditividad numerable, la intersección $\bigcap_{i=1}^{\infty}\left[A_{1}^{i}+A_{2}^{i}+\cdots+A_{k_{i}}^{i}+\cdots\right]$ sería no numerable para cualquier sucesión de enteros positivos $\left\{k_{i}\right\}$.

Banach y Kuratowski muestran a su vez que la hipótesis del continuo permite construir la doble sucesión $\left\{A_{k}^{n}\right\}$ pero que esta es tal que la intersección $\bigcap_{i=1}^{\infty}\left[A_{1}^{i}+A_{2}^{i}+A_{3}^{i}+\cdots\right]$ es a lo más numerable. Es esta incompatibilidad la que lleva a los autores a enunciar la frase que citamos anteriormente.

A partir de la demostración presentada por Banach y Kuratowski se puede concluir que la condición de aditividad numerable lleva a la necesidad de que el número cardinal del conjunto en el cual se va a definir la medida sea mayor que $\aleph_{1}$ si bien no ha sido explícita la incompatibilidad de la condición con el hecho de que la cardinalidad de E sea precisamente $\boldsymbol{\aleph}_{1}$.

En 1930 Banach, Tarski y Ulam publican resultados precisamente sobre este punto.

Banach (1930) establece una condición necesaria para la existencia de una medida que cumpla las tres condiciones establecidas en Banach y Kuratowski (1929) y se muestra de manera concluyente que la posibilidad de definir una medida en un conjunto depende de su número cardinal. En efecto, para poder definir una medida sobre un conjunto, el número cardinal de este debe ser inaccesible. $^{43}$

Para hacer ver esto, Banach hace dos cambios con respecto a los supuestos del artículo de 1929; la hipótesis del continuo es remplazada por la hipótesis generalizada del continuo, es decir que $2^{\aleph_{\alpha}}=\aleph_{\alpha+1}$ para todo ordinal $\alpha$, y el segundo cambio tiene que ver con la condición de aditividad numerable. Esta condición es generalizada para cardinales más allá de $\aleph_{0}$. Banach presenta la siguiente definición: Una medida $m$ es $\boldsymbol{\aleph}_{\alpha}$ - aditiva si dada una sucesión $\left\{G_{\gamma}\right\}_{\gamma<\omega_{\alpha}}$ de conjuntos ajenos entre sí se tiene que $m\left(\bigcup_{\gamma<\omega_{\alpha}} G_{\gamma}\right)=\sum_{\gamma<\omega_{\alpha}} m\left(G_{\gamma}\right)$. Una medida es de tipo $\boldsymbol{\aleph}_{\alpha}$ si es $\boldsymbol{\aleph}_{\beta}$ - aditiva para cada $\boldsymbol{\aleph}_{\beta}<\boldsymbol{\aleph}_{\alpha}$ pero no para $\boldsymbol{\aleph}_{\alpha}$.

Con base en estas hipótesis y nuevas definiciones Banach prueba el siguiente resultado: Si sobre un conjunto $\mathrm{E}$ es posible definir una medida de tipo $\boldsymbol{\aleph}_{\alpha}$, entonces $\boldsymbol{\aleph}_{\alpha}$ es un cardinal inaccesible.

Parecería natural pensar que las hipótesis más fuertes jugarían un papel equivalente a las previas en este nuevo resultado; sin embrago, es el propio Banach quien señala la importancia de una nueva hipótesis: la existencia de los cardinales inaccesibles.

Esta demostración de Banach tiene como objetivo establecer una condición necesaria bajo la cual el problema de la medida tenga solución y a su vez mostrar que la hipótesis del continuo implica que el intervalo unitario, o cualquier conjunto de cardinalidad $2^{\aleph_{0}}=\aleph_{1}$ no puede aceptar una medida que satisfaga la condición de aditividad numerable.

${ }^{43}$ Se dice que un cardinal $\boldsymbol{\aleph}$ es inaccesible si es mayor que $\boldsymbol{\aleph}_{0}$, es regular y $2^{\lambda}<x$ para todo $\lambda<x$. 
Como corolario a este teorema, Banach prueba que el número cardinal de un conjunto debe ser mayor que el primer cardinal inaccesible para que en el conjunto se pueda definir una medida que sea numerablemente aditiva. $\mathrm{O}$, dicho en otras palabras: Si $\kappa$ es el primer cardinal inaccesible, $\kappa>\boldsymbol{\aleph}_{0}$, y el número cardinal de un conjunto $\mathrm{E}$ es igual a $\lambda<\kappa$, entonces, si existe una medida en $\mathrm{E}$, esta solo puede ser finitamente aditiva. De esta manera, el continuo es demasiado pequeño para aceptar una medida numerablemente aditiva.

Dadas las nuevas hipótesis utilizadas por Banach en este artículo creemos que es importante aclarar que no hay ninguna relación entre el axioma de elección y la hipótesis del continuo, o más en general entre el axioma de elección y la determinación precisa de la cardinalidad del continuo. Gödel (1940) mostró que la negación de la hipótesis del continuo es consistente con ZFC y el resultado de Cohen (1963) muestra que la hipótesis del continuo de hecho es independiente de ZFC. En esta misma línea consideramos importante subrayar que, aunque no hay relación entre el axioma de elección y la hipótesis del continuo, Sierpinski (1947) demuestra que la hipótesis generalizada del continuo usada por Banach en este artículo implica el axioma de elección. Los detalles de esa demostración quedan fuera del ámbito de este artículo, simplemente queremos acentuar la relación profunda que existe entre estos enunciados.

Ahora bien, también en 1930, Tarski publica un artículo llamado "Une contribution à la théorie de la mesure" (Tarski 1930), en el cual plantea la siguiente pregunta: ¿Dado un conjunto infinito $\mathrm{E}$, existe una función $m(\mathrm{X})$ que haga corresponder a cada subconjunto $\mathrm{X}$ de $\mathrm{E}$ un número real no negativo de manera que (1) $m(X)$ sea aditiva en el sentido restringido, (2) $m(X)$ se anule para todo subconjunto $\mathrm{X}$ de $\mathrm{E}$ que esté compuesto de un solo punto y (3) $m(\mathrm{X})$ no sea idénticamente cero?

La respuesta que Tarski da a este problema (que llama generalización del problema amplio de la medida) es la siguiente: "Yo voy a probar aquí con la ayuda del axioma de elección que el problema enunciado hace un momento posee una solución positiva; voy a mostrar además que se puede determinar la función $m(X)$ de manera que ella solo admita dos valores: 0 y 1" (Tarski 1930, p. 43).

Después de la publicación de estos artículos surge la pregunta de si las hipótesis utilizadas pueden ser sustituidas por condiciones más débiles. Banach había mostrado ya que el tamaño de un conjunto está fuertemente relacionado con las propiedades de las medidas que sobre él se pueden definir y surge así la pregunta sobre la relación específica que existe entre el número cardinal de un conjunto y la condición de aditividad que satisfacen las medidas definidas en él.

Es en este mismo año cuando Stanislaw Ulam, en "Zur Masstheorie in der allgemeinen Mengenlehre" (Ulam 1930), da respuesta a estas cuestiones. Ulam demuestra que si $\mathrm{E}$ es un conjunto con número cardinal $|\mathrm{E}| \mathrm{y}$ no existe un cardinal inaccesible $\kappa$ tal que $\kappa<|E|$, entonces $|E|$ no es un cardinal medible. ${ }^{44}$ Esto

\footnotetext{
${ }^{44}$ Existen varias definiciones (equivalentes) de lo que significa que un cardinal sea medible; consideramos que la más accesible es la siguiente: Un cardinal no numerable $\kappa$ es medible si existe una medida no trivial, $\kappa$-aditiva y que toma únicamente los valores 0 y 1 definida sobre el conjunto potencia de $\kappa$.
} 
implica que sobre el conjunto E no se puede definir una medida que satisfaga las condiciones 1-3 del problema planteado en 1929 por Banach y Kuratowski (Banach \& Kuratowski 1929).

Este resultado a su vez implica que una medida $m$ definida sobre todos los subconjuntos de un conjunto $\mathrm{X}$ de potencia $\boldsymbol{\aleph}_{1}$ es idénticamente cero si es cero en todos los subconjuntos que constan de un solo elemento. Para poder aplicar este resultado a medidas en el continuo es necesario hacer alguna suposición sobre la cardinalidad $\mathfrak{c}$ del continuo: si se supone la hipótesis del continuo, o una hipótesis un poco más suave, a saber que ningún cardinal menor o igual a $\mathfrak{c}$ es débilmente inaccesible, ${ }^{45}$ entonces se puede concluir que una medida finita definida sobre todos los subconjuntos de un conjunto de cardinalidad $\mathfrak{r}$ es idénticamente cero si vale cero en los conjuntos formados por un solo punto.

Este artículo de Ulam permite cerrar este ciclo de artículos que vinculan de manera intrínseca el problema de la medida con el axioma de elección y es fácil ver, a partir de estos artículos, que fue únicamente cuando Banach, Kuratowski, Tarski y Ulam se dieron cuenta de que una condición necesaria que debe satisfacer el conjunto para ser medido está vinculada a su número cardinal que se pudo vislumbrar la relación tan estrecha que guardan los axiomas de la teoría de conjuntos con el problema de la medida.

\section{Conclusión}

El objetivo de este artículo, como mencionamos en la introducción, ha sido estudiar la relación que guardan entre sí el axioma de elección y la teoría de la medida y en particular el papel que juega dicho axioma para comprender esta teoría matemática. Para lograr esto hemos expuesto un capítulo poco conocido de la historia del axioma de elección que a su vez nos ha mostrado la necesidad de colocar el estudio sobre dicho axioma en un plano filosóficamente distinto. Tradicionalmente, se habían llevado a cabo dos tipos de estudios en torno de este axioma, el primero de carácter lógico relacionado con su consistencia (demostrada por Gödel en 1939) y su independencia (demostrada por Cohen en 1963) y el segundo, más delicado, en un plano relacionado con su carácter o su legitimidad, comparándolo con los otros axiomas de la teoría de conjuntos, analizando su naturaleza e inclusive su veracidad (como lo hace Russell 1911, 1927, pp. 299-30046 o Bernays $1935^{47}$ por ejemplo). Nuestro texto intenta contribuir a que la discu-

\footnotetext{
${ }^{45}$ Se dice que un cardinal no numerable es débilmente inaccesible si es un cardinal límite y es regular.

${ }^{46}$ En estos textos, entre otros, Russell presenta su postura frente a la veracidad del axioma de elección que, como es bien sabido, cambió radicalmente a través de los años. En 1911 Russell afirma que en su "opinión no hay ninguna razón para creer que el axioma [...] sea verdadero” (Russell 1911, p. 501) y años más tarde, en 1927, afirma que ha "sido llevado por los argumentos del Dr. H. M. Sheffer y del Sr. F. P. Ramsey a ver que el axioma de Zermelo es verdadero" (Russell 1927, pp. 299-300).

${ }^{47}$ Notamos que la posición que toma Bernays en este artículo difiere de las de Russell que mencionamos y por eso nos parece pertinente mencionarla como ejemplo aquí, para presentar un espectro amplio de las actitudes frente a este axioma (si bien es cierto que la de Bernays se podría considerar como más cercana a la contemporánea).
} 
sión en torno de este axioma se plantee en otro terreno y esto nos ha permitido abrir la puerta a la discusión sobre la comprensión en matemáticas dentro del debate contemporáneo de la filosofía de la práctica matemática.

En el caso que hemos estudiado a lo largo de este artículo cabe mencionar que lo que la historia nos ha mostrado de manera clara es que el axioma de elección juega un papel central y puntual para poder comprender la teoría de la medida. No obstante, es importante subrayar que el axioma de elección, si bien permite un entendimiento más profundo de dicha teoría, no permite por sí solo la comprensión total de la teoría. Nuestro artículo no pretende reducir la comprensión de la teoría de la medida a un único axioma: es claro que hay cuestiones que el axioma no puede explicar, como por ejemplo las diferentes soluciones que los diversos problemas de la medida admiten al variar la dimensión (más no la cardinalidad) del espacio en cuestión. Es así que nuestro artículo deja abierta la pregunta de qué otros ingredientes se necesitan para comprender plenamente la teoría de la medida; este será el tema de un artículo posterior.

Bibliografía

Álvarez, C. (1993), "Sur l'origine de l'hypothèse du continu", Sciences et techniques en perspective 26: 250-273.

Baire, R. (1990), Euvres Scientifiques, Paris: Gauthier Villars.

Banach, S. (1923), "Sur le problème de la mesure", Fundamenta Mathematicae 4: 7-33.

Banach, S. (1930), "Über additive Massfunktionen in abstrakten Mengen", Fundamenta Mathematicae 15: 97-101.

Banach, S. y C. Kuratowski (1929), "Sur une généralisation du problème de la mesure", Fundamenta Mathematicae 14: 127-131.

Banach, S. y A. Tarski (1924), "Sur la décomposition des ensembles de points en parties respectivement congruentes”, Fundamenta Mathematicae 6: 244-277.

Benacerraf, P. y H. Putnam (eds.) (1983), Philosophy of Mathematics: Selected Readings, New York: Cambridge University Press.

Bernays, P. (1935), "Sur le platonisme dans les mathématiques”, L’Enseignement Mathématique 34: 52-69. (Versión inglesa: "On Platonism in Mathematics”, en Benacerraf \& Putnam (1983), pp. 258-271.)

Bernstein, F. (1901), Untersuchungen aus der Mengenlehre, Tesis Doctoral, Göttingen.

Borel, E. (1895), "Sur quelques points de la théorie des fonctions", Annales Scientifiques de l'Ecole Normale Supérieure 3e série 12: 9-55.

Borel, E. (1898), Leçons sur la Théorie des Fonctions, Paris: Gauthier-Villars.

Borel, E. (1904), "Quelques remarques sur les principes de la théorie des ensembles", Mathematische Annalen 59: 514-516. 
Borel, E. (1905), Leçons sur les fonctions de variables réelles, Paris: Gauthier-Villars.

Burstin, C. (1914), "Eigenschaften meßbarer und nichtmeßbarer Mengen", Sitzungsber. Der Akad. Der Wiss. In Wien, Math.-Nat. Klasse 123(Abt. Ila): 1525-1551.

Cantor, G. (1878), "Ein Beitrag zur Mannigfaltigkeitslehre", Journal für die reine und angewandte Mathematik 84: 242-258.

Cantor, G. (1883), "Über unendliche, lineare Punktmannichfaltigkeiten 5", Mathematische Annalen 21: 545-591.

Cantor, G. (1884b), "De la puissance des ensembles parfaits de points", Acta Mathematica 4: 381-392.

Cantor, G. (1884a), "Über unendliche lineare Punktmannigfaltigkeiten 6”, Mathematische Annalen 23: 453-488.

Cantor, G. (1895), "Beiträge zer Begründung der transfiniten Mengenlehre (Erster Artikel)", Mathematische Annalen 46: 481-512.

Cantor, G. (1932), Gesammelte Abhandlungen mathematischen und philosophischen Inhalts, Zermelo, E. (ed.), Berlin: Springer.

Cantor, G. (2006), Fundamentos para una teoría general de conjuntos, en Ferreirós, J. (ed.), Madrid: Crítica.

Cavaillès, J. (1981), Méthode Axiomatique et Formalisme, Paris : Hermann.

Cavaillès, J. (1994), Oeuvres Complètes de Philosophie des Sciences, Paris: Hermann.

Cohen, P. J. (1963), "The Independence of the Continuum Hypothesis", Proceedings of the National Academy of Sciences of the United States of America 50: 1143-1148.

Cohen, P. J. (1964), "The Independence of the Continuum Hypothesis II", Proceedings of the National Academy of Sciences of the United States of America 51: 105-110.

Czyz, J. (1994), Paradoxes of Measures and Dimensions Originating in Felix Hausdorff's Ideas, London: World Scientific Publishing Co.

Denjoy, A. (1946), L'énumération transfinie. Livre I. La notion du rang, Paris : Gauthier-Villars.

du Bois Reymond, P. (1887), Théorie générale des fonctions, Nice: Première partie.

Fraenkel, A. y Y. Bar-Hillel (1958), "Foundations of Set Theory", Amsterdam: North-Holland Publishing Co.

Frege, G. (1893), Grundgesetze der Arithmetik, begriffsschruftlich abgeleitet, Vol. 1, Jena: Hermann Pohle.

Frege, G. (1903), Grundgesetze der Arithmetik, begriffsschruftlich abgeleitet, Vol. 2, Jena: Hermann Pohle.

Frege G. (1976), Wissenschaftlicher Briefwechsel, en Gabriel, G., Hermes, H., Kambartel, F., Thiel, T. y A. Veraart (eds.), Hamburg: Feliz Meiner.

Gödel, K. (1940), The Consistency of the Axiom of Choice and of the Generalized Continuum Hypothesis with the Axioms of Set Theory, Princeton: Princeton University Press.

Gödel, K. (1947), “What is Cantor's continuum problem?”, American Mathematical Monthly 54: 515-525.

Hadamard, J. (1905), "Cinq lettres sur la théorie des ensembles", Bulletin de la Société Mathématique de France 33: 261-273. 
Hausdorff, F. (1914), Grundzüge der Mengenlehre, Leipzig: W. de Gruyter.

Hawkins, T. (1975), Lebesgue's Theory of Integration, New York: Chelsea Publishing Company.

Hilbert, D. (1900), "Mathematische Probleme", Nachrichten von der Königlichen Gesellschaft der Wissenschaften zu Göttingen, mathematisch-physikalische Klasse: 253-297.

Hilbert, D. (1905), Logische Principen des mathematischen Denkens, Library of Mathematisches Seminar, Göttingen: Universidad de Göttingen.

Heine, E. (1872), "Elemente der Functionenlehre", Journal für die reine und angewandte Mathematik 74: 172-188.

Herrlich, H. (2006), Axiom of Choice, Berlin: Springer Verlag.

Husserl, E. (1979), Aufsätze und Rezensionen (1890-1910), en Rang, B. (ed.), Husserliana vol. XXII, Netherlands: Martinus Nijhoff.

Jech, T. (1973), The Axiom of Choice, Amsterdam: North Holland Publishing Co.

Jech, T. (1978), Set Theory, New York: Academic Press.

Jordan, C. (1892), "Remarques sur les intégrales définies", Journal de Mathématiques pures et appliquées 4(8): 69-99.

König, J. (1905), “Zum Kontinuum-Problem”, Mathematische Annalen 60: 177-180.

Kowalewski, G. (1950), Bestand und Wandel. Meine Lebenserinnerungen. Zugleich ein Beitrag zur neueren Geschichte der Mathematik, München: Oldenbourg.

Kuratowski, K. y A. Mostowski (1968), Set Theory, Amsterdam: North Holland Publ. Co.

Lebesgue, H. (1902), "Intégrale, longueur, aire”, Annali di Mathematica III(7): 31-129.

Lebesgue, H. (1904), Leçons sur l'Intégration et la Recherche des Fonctions Primitives, Paris: Gauthier-Villars.

Lebesgue, H. (1918), "Remarques sur le Théories de la Mesure et de l'Intégration", Annales de l'Ecole Normale Supérieure III(35): 191-250.

Lebesgue, H. (1971), “A propos de quelques travaux mathématiques récents”, L'Enseignement Mathématique XVII(1): 1-48.

Lebesgue, H. (1972), Oeuvres Scientifiques, 5 vols., Genèvre: L'Enseignement Mathématique.

Lévy, P. (1950), “Axiome de Zermelo et nombres transfinis”, Annales Scientifiques de l'Ecole Normale Supérieure, 3a serie, 67: 15-49.

Michel, A. (1992), Constitution de la Théorie Moderne de l'Intégration, Paris: Vrin.

Moore, G. (1982), Zermelo's Axiom of Choice, Its Origins, Development and Influence, New York: Springer Verlag.

Moore, G. (2002), "Hilbert on the Infinite: The Role of Set Theory in the Evolution of Hilbert's Thought", Historia Mathematica 29(1): 40-64.

Peano, G. (1890), "Démonstration de l'intégrabilité des équations différentielles ordinaires", Mathematische Annalen, pp. 182-228.

Purkert, W. y H. J. Ilgauds (1987), Georg Cantor 1845-1918, Basel : Birkhäuser.

Russell, B. (1911), "Sur les axiomes de l'infini et du transfini", Bull. Soc. Math. France 39: 488-501.

Russell, B. (1927), The Analysis of Matter, London: Allen and Unwin. 
Sierpinski, W. (1918), "L'axiome de M. Zermelo et son rôle dans la théorie des ensembles et l'analyse", Bulletin de l'Académie des Sciences de Cracovie, Classe des Sciences Math., Série A, pp. 97-152.

Sierpinski, W. (1934), “L’Hypothése du Continu”, Monografje Matematyczne, Vol. 4, Warsawa-Lwów.

Sierpinski, W. (1947), "L'hypothèse du continu et l'axiome du choix”, Fundamenta Mathematicae 34: 1-5.

Sierpinski, W. (1974), Oeuvres Choisies, 3 Vols., Warszava: PWN Editions Scientifiques de Pologne.

Solovay, R. (1970), "A Model of Set Theory in which every Set of Reals is Lebesgue Measurable”, Annals of Mathematics, 2a serie, 92: 1-56.

Tannery, J. (1897), Introduction à la théorie des fonctions d'une variable, Paris : Hermann.

Tarski, A. (1930), "Une contribution à la théorie de la mesure", Fundamenta Mathematicae 15: $42-50$.

Ulam, S. (1930), "Zur Masstheorie in der allgemeinen Mengenlehre", Fundamenta Mathematicae 16: $140-150$.

Ulam, S. (1943), "What is Measure?", American Mathematical Monthly 50(10): 597-602.

van Heijenoort, J. (1967), From Frege to Gödel, A source book in mathematical logic, 1879 1931, Cambridge: Harvard University Press.

Vitali, G. (1905), Sul problema della misura dei gruppi di punti di una retta, Bologna: Tip. Gamberini e Parmeggiani.

Zermelo, E. (1904), "Beweis, dass jede Menge wohlgeordnet werden kann”, Mathematische Annalen 59: 514-516.

Zermelo, E. (1908b), "Untersuchungen über die Grundalgen der Mengenlehre. I”, Mathematische Annalen 65: 261-281.

Zermelo, E. (1908a), "Neuer Beweis für die Möglichkeit einer Wohlordnung”, Mathematische Annalen 65: 107-128. 